\title{
Asymptotic, convergent, and exact truncating series solutions of the linear shallow water equations for channels with power law geometry*
}

\author{
Geir Pedersen ${ }^{\dagger}$
}

\begin{abstract}
The present study was originally motivated by some intriguing exact solutions for waves propagating in nonuniform media. In particular, for special depth profiles reflected waves did not appear and ray theory became exact. Herein, geometrical optics is employed to obtain asymptotic series for waves of general shapes in non-uniform narrow channels, within the framework of linear shallow water theory. While being kept simple, the series incorporate higher order contributions that may describe the evolution of waves with high accuracy. The higher orders also contain a secondary wave system. For selected classes of geometries and wave shapes explicit solutions are calculated and compared to numerical solutions. Apart from the vicinity of shorelines, say, higher order expansions generally may provide very accurate approximations to the full solutions. The asymptotic series are analyzed for different wave shapes and are found to be convergent for cases where the basic wave profiles have compact support (finite length). A number of new, closed form, exact solutions are also found. The asymptotic expansion is put into a context by employing it for the transmission of waves from a uniform channel section into a nonuniform one. Additional results and side topics are presented in a supplement.
\end{abstract}

Key words. Ray theory, asymptotic expansions, shallow water waves, channels

AMS subject classifications. 68Q25, 68R10, 68U05

1. Introduction. For wave propagation in uniform media solutions are available as uniform, harmonic wave trains. When the the medium is slowly varying such solutions may be generalized to a slowly varying wave train by means of ray theory. Variants of ray theory are employed in a vast number of articles and are included in most textbooks on waves. Such theories may be developed by direct invocation of dispersion relation and energy expressions, by perturbation schemes, most notably the WKB method, [19, 13, 2, 4] or a variational approach [35]. Ray theory is a useful vehicle for the conception of wave phenomena such as focusing, amplification, wave trapping and shadowing. It is important for understanding the behaviour of water waves in a bathymetry and propagation of acoustic and seismic waves in the layered atmosphere and earth, respectively $[27,1]$. On the other hand, standard ray theory has severe limitations concerning quantitative description of waves in even moderately complex conditions due to singularities at caustics and lack of diffraction. Ray theory is mostly applied to linear harmonic modes which then may be combined in a Fourier integral to describe waves of more general shapes. There are a few examples of similar approaches applied to specific nonlinear wave forms, such as shocks [34, 35, 3] and solitary waves [14, 20, 16, 17, 22].

In shallow water theory waves of normal incidence on laterally uniform beaches lead to mathematical formulations with one spatial dimension in addition to time. The depth may then be written $h(x)$ where $x$ is the coordinate along an axis parallel to the direction of

\footnotetext{
*February 3, 2021

${ }^{\dagger}$ Department of Mathematics, University of Oslo, PO box 1053, 0316 Oslo, Norway (geirkp@math.uio.no, https:// www.mn.uio.no/math/english/).
} 
wave advance. Some depth profiles then yield equations of standard types with analytic solutions. For an inclined plane $(h=\alpha x)$ periodic waves may be expressed in terms of Bessel functions. In this case the standard WKB solution, equivalent to asymptotic expansion of the Bessel functions, predicts that the amplitude is proportional to $x^{-\frac{1}{4}}$. While the inclined plane generally is conceived as the most basic one, other configurations are simpler and allow for a more transparent analysis, in particular for the linear case. Simple exact solutions are known for the $h=\alpha x^{\frac{4}{3}}$ profile for which the surface elevation evolves according to ray theory. [33] exploited this to obtain explicit solutions for waves generated by slides on a slope, in a corresponding form as in constant depth. Later, [10] retrieved the $h=\alpha x^{\frac{4}{3}}$ profile as the only one where ray theory for periodic waves gave an exact result for the surface elevation. Then the exact solution for the surface elevation for a wave of any form followed.

Linearized shallow water theory for long waves in narrow channels also leads to equations in one spatial dimension for the surface elevation and the longitudinal velocity, while the geometry may be described in terms of the channel width and the effective depth, which will be defined later. By inclusion of finite amplitude and non-hydrostatic effects these may be generalized to Boussinesq or KdV type equations [26, 25, 28, 29].

Within linear shallow water theory there is a particularly simple exact solution for which changing widths and depths of a channel counterbalances to allow wave propagation without amplification. Inclined channels with parabolic cross sections allow for other exact solutions $[5,6,7]$. For this case the linear solution has a similar structure as for the plane, normal incidence, case with the $h=\alpha x^{\frac{4}{3}}$ profile. Furthermore, [8] applied the WKB method to the class of channels for which both the transverse shape and lengthwise slope were defined by power functions in $x$. They found a set of combinations of shapes and slopes, including those mentioned above, that made the expression for the surface elevation exact. Then, surface excursions with finite wavelength can propagate without the evolution of a secondary system of surface elevations. This may seem to indicate absence of any form of diffraction or reflection. However, these surface elevations come with velocity fields that have modified shapes relative to the ray solution and a trailing region of constant volume flux. The cases where ray theory is exact can be conceived as cases for which the variable coefficient equations may be transformed to a standard wave equation with constant coefficients. A slightly more general approach transforms the shallow water equations to a constant coefficient Klein-Gordon equation [15, $9,24]$. However, this and a few other exact solutions of the shallow water equations with non-constant depth, such as the oscillations in parabolic basins [32], are less closely related to the present work.

The first objective of the present article is to obtain a wider perspective on the exact ray solutions from the literature. Are they unique with properties that are distinct in relation to solutions which cannot be exactly expressed in this manner? Higher order expansions are designed for investigation of the cases where low orders do not provide exact solutions. Since the medium is non-dispersive we employ a direct formulation of ray theory for general wave shapes; notably single-crested pulses. This is preferred over application through the Fourier transform, which, in the present case, is a cumbersome detour at best. To obtain solutions that are quite explicit, and hence open to detailed analysis and interpretation, a selection of particular channel geometries and wave shapes is made. This include also cases where uniform 
and non-uniform channel geometries are joined and where the transmission and reflection at the junction may be analyzed by employing the asymptotic expansions. The validity and accuracy of the asymptotic solutions are checked through comparison with numerical solutions and by the derivation of a theoretical error constraint. In the supplement additional details, solutions and plots are presented, as well as secondary topics such as conservation properties and implications of the transmission properties for calculation of runup on a beach.

2. Formulation. Exempting curved channels from this study we introduce the coordinates $x, y$ and $z$ in the longitudinal, transverse and vertical directions respectively. A channel geometry is defined in figure 1 . The depth is expressed as $h(x, y)$, while the equilibrium width at the surface is $B$. The surface elevation and longitudinal velocity are denoted by $\eta$ and $u$, respectively. Dimensionless expressions are used throughout this article. The depth scale is $h_{c}$ and a characteristic length $x_{c}$, associated with the change of the geometry, is used for scaling $x$ in the hydrodynamic equations. With $g$ as the acceleration of gravity the time scale is $x_{c} / \sqrt{g h_{c}}$ and the ratio between the surface and velocity becomes $\eta_{c} / u_{c}=\sqrt{h_{c} / g}$. When convenient, the scaling may give a reference position $x_{0}=1$ at $t=0$ with $B\left(x_{0}\right)=\bar{h}\left(x_{0}\right)=1$ and, say, a unitary amplitude. A shorter spatial scale, $x_{c} / \kappa$, where $\kappa$ is large, will be used for wave lengths.

2.1. Linear shallow water waves in a channel. The equations are described in, for instance [18] $\S 185$. Within the shallow water approximation $\eta$ and $u$ are uniform in the span-wise directions. In the linear approximation the surface width, $B$, is regarded as fixed in time. It is convenient to introduce the effective depth, $\bar{h}$, which is defined as the equilibrium cross section area, divided to $B$. For the dynamic cross-section area we then find $\bar{h} B+B \eta$ and the momentum and continuity equations may be expressed according to

$$
u_{t}=-\eta_{x}, \quad \eta_{t}=-\frac{1}{B}(B \bar{h} u)_{x}
$$

where indices indicate differentiation. Elimination of $u$ yields

$$
\eta_{t t}=\frac{1}{B}\left(B \bar{h} \eta_{x}\right)_{x}=\frac{B_{x}}{B} \bar{h} \eta_{x}+\left(\bar{h} \eta_{x}\right)_{x}
$$

When $B$ is constant we retrieve the standard shallow water equations for the plane case. For a rectangular channel $B$ and $\bar{h}$ are the width and equilibrium depth of the channel, respectively. It is noteworthy that channels of any shape correspond to a rectangular one, with depth equal to $\bar{h}$ and width $B$, by means of (2.1) or (2.2). Numerical solution of (2.2) is quite straightforward and is briefly discussed in section SM5.1. However, a proper comparison with the asymptotic solutions may require a high resolution.

3. The ray expansion. Usually ray and transport equations are formulated for harmonic modes. The evolution of a more general wave form may then be found by inversion of Fourier type integrals. However, such inversions are cumbersome. Herein we exploit the lack of dispersion for shallow water waves to employ a method that is directly applicable to waves of any shape, while it also extends to higher orders. In general, such an approach may not be applied to dispersive and nonlinear waves. 


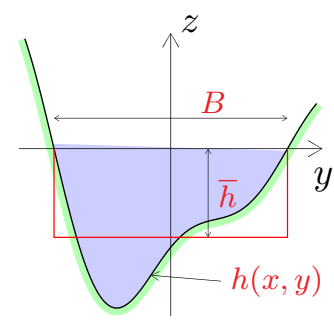

Figure 1. Definition sketch. Cross section of a generic channel. The side walls and bottom of the equivalent rectangular channel is indicated by the thin vertical and horizontal lines.

3.1. Leading order asymptotic approximation in a channel. Waves in slowly varying channels may approximately retain their shape, while the amplitude and length change. From [18] $\S 185$ a leading order approximation is adapted as

$$
\eta=A(x) F(\Theta), \quad u=\mp \bar{h}^{-\frac{1}{2}} A(x) F(\Theta),
$$

where $F$ is an arbitrary shape function and

$$
\Theta=\kappa(t \pm \tau), \quad \tau=\int_{x_{0}}^{x} \bar{h}^{-\frac{1}{2}} d \hat{x}, \quad A=\sigma B^{-\frac{1}{2}} \bar{h}^{-\frac{1}{4}} .
$$

Here, $\tau$ is the propagation time from a reference position $x_{0}$ with the local wave speed $c_{0}=\sqrt{h}$, while the identical appearance of $t$ in $\Theta$ is due to the invariance of the wave period. The relation for $A$ in terms of $B$ and $\bar{h}$ follows from energy conservation and is often referred to as Green's law. When $B \bar{h}^{\frac{1}{2}}$ is decreasing in the direction of wave advance the wave is amplified. In the opposite case the amplitude attenuates. The constant $\sigma$ can be made equal to unity by the appropriate scaling (see section 2), but is kept in the relations for now. The factor $\kappa$ governs the wavelength and a large value makes the wave short in comparison to the length scales of the geometry.

The relations (3.1) will be reproduced in the derivation of higher order theory. Then, $A F$, renamed to $A_{0} F_{0}$, is the leading order approximation for $\eta$ and will be referred to as the principal wave.

3.2. Higher order asymptotic approximations. With $A$ and $F$ renamed to $A_{0}$ and $F_{0}$, respectively, a higher order approximation may be expressed as an asymptotic series on the form

$$
\eta \sim A_{0}(x) F_{0}(\Theta)+\kappa^{-1} A_{1}(x) F_{1}(\Theta)+\kappa^{-2} A_{2}(x) F_{2}(\Theta) \ldots
$$

Here $\kappa$ is used as an ordering parameter, but the magnitudes of terms could have been made equally transparent by observing the number of derivatives through $F_{j}$ in relation to derivatives of $\bar{h}, B, A_{j}$ etc. The expansion (3.3) is a special case of geometrical optics as defined in [34] $\S$ 7.7. If harmonic shapes, $F_{j}=(-i)^{j} \exp (i \Theta)$, are used (3.3) will become the standard WKB 
technique. However, herein we will study other shapes. The asymptotic series may then have quite different properties than that of the WKB expansion.

Substituting (3.3) into the single PDE for $\eta,(2.2)$, and sorting by magnitude we obtain

$$
0=\kappa\left[\mp\left(A_{0} B\right)^{-1}\left(B \bar{h}^{\frac{1}{2}} A_{0}^{2}\right)_{x} F_{0}^{\prime}\right]+\kappa^{0}\left[-B^{-1}\left(B \bar{h} A_{0, x}\right)_{x} F_{0} \mp\left(A_{1} B\right)^{-1}\left(B \bar{h}^{\frac{1}{2}} A_{1}^{2}\right)_{x} F_{1}^{\prime}\right]+\ldots
$$

where indices after commas indicate differentiation. The $O\left(\kappa^{2}\right)$ part vanishes due to $\tau_{x}=$ $\pm \bar{h}^{-\frac{1}{2}}$, wheras the $O(\kappa)$ balance reproduces the leading order approximation

$$
A_{0}=\sigma B^{-\frac{1}{2}} \bar{h}^{-\frac{1}{4}}
$$

From $O\left(\kappa^{0}\right)$ we have a balance between terms with factors from two subsequent orders in (3.3). Since $\Theta$ and $x$ must be treated as independent variables these orders are annihilated by the recursion $(j=0,1 \ldots)$

$$
F_{j+1}(\Theta)=\int_{-\infty}^{\Theta} F_{j}(\hat{\Theta}) d \hat{\Theta}, \quad A_{j+1}^{-1}\left(B \bar{h}^{\frac{1}{2}} A_{j+1}^{2}\right)_{x}=\mp\left(B \bar{h} A_{j, x}\right)_{x} .
$$

When wave propagation to the left $(\Theta=\kappa(\tau+t))$ is assumed for simplicity (3.4) may be used with (3.5) to give the explicit amplitude recursion

$$
A_{j+1}=-\frac{1}{2} \bar{h}^{\frac{1}{2}} A_{j, x}+\frac{1}{2} \sigma^{-2} A_{0} \int_{x_{r}}^{x} \bar{h} B A_{0, x} A_{j, x} d \hat{x} .
$$

The reference position, $x_{r}$, may be chosen freely for each $j$. Changing the value of an $x_{r}$ corresponds to adding a constant times $A_{0}$ to $A_{j}$. This ambiguity corresponds to a redefinition of the principal wave at each level in the expansion and is exploited in section 5 .

Employing both equations in (2.1) we find the velocity

$$
u \sim-\left[\bar{h}^{-\frac{1}{2}} A_{0} F_{0}(\Theta)+\kappa^{-1}\left(\bar{h}^{-\frac{1}{2}} A_{1}+A_{0, x}\right) F_{1}(\Theta)+\kappa^{-2}\left(\bar{h}^{-\frac{1}{2}} A_{2}+A_{1, x}\right) F_{2}(\Theta) \ldots\right]+Q_{f}(B \bar{h})^{-1},
$$

where $Q_{f}$ is a constant. On its own, the last term, combined with $\eta=0$, is a solution of the linear shallow water equations. Hence, it is unrelated to the principal wave and yields a volume flux, of constant strength, along the channel. Accordingly, we set $Q_{f}=0$. The omitted term should not be confused with the similar, but local, velocity field in section 4.2.

It is now assumed that the principal wave, defined through $F_{0}$ has compact support in the sense that $F_{0}$ is zero for $\Theta<\Theta_{0}$ and $\Theta>\Theta_{L}$. If the total integral of $F_{0}$ is nonzero, as for a principal wave of elevation, then $F_{1}$ is constant for $\Theta>\Theta_{L}$, while $F_{j}$ is a polynomial of degree $j-1$. When $F_{0}$ instead decays exponentially for large $\Theta$ the $F_{j}$ functions still have a dominant polynomial behaviour for large $\Theta$. Hence, there is a a trailing system with surface excursion and fluid velocity behind the principal wave. The trailing system is needed to balance the volume and momentum in the principal wave and does also drain energy from it (details in sections SM7, SM2.4). Similar trailing systems are described for solitary waves that shed volume due to geometry or lateral non-uniformity of the crest [16, 17, 22]. 
3.3. Waves propagating in the positive $x$-direction. When waves are propagating toward increasing $x$ an asymptotic expression may be readily deduced from (3.2), (3.3), (3.5) and the momentum equation in (2.1)

$$
\eta \sim \sum_{j=0}^{\infty}(-1)^{j} \kappa^{-j} A_{j} F_{j}(\hat{\Theta}), \quad u \sim \bar{h}^{-\frac{1}{2}} A_{0} F_{0}(\hat{\Theta})+\sum_{j=1}^{\infty}(-1)^{j} \kappa^{-j}\left(\bar{h}^{-\frac{1}{2}} A_{j}-A_{j, x}\right) F_{j}(\hat{\Theta})
$$

where all quantities, except the modified phase $\hat{\Theta}=\kappa(t-\tau)$, are defined as previously.

4. Solutions for selected profiles. Explicit expressions for the terms in (3.3) may only be obtained for selected classes of geometries and principal wave shapes. Geometries defined by single power functions or exponentials yield simple recursions for the amplitudes $A_{0}, A_{1}$ etc. When $\bar{h}$ is monotonic it can be used as spatial variable and (3.6) rewritten accordingly. Then, certain relations between $\frac{d \bar{h}}{d x}$ and $\bar{h}$ yield new explicit recursions for the amplitudes, in addition to those mentioned above. For brevity, only the power function geometries are analyzed herein. The other options are briefly outlined in the sections SM3.4 and SM6 of the supplement.

Two types of principal wave shapes are employed; one where $\eta$ tapers off exponentially at the outskirts and another one with compact support (strictly finite length). There are some striking differences concerning the asymptotic series for these two groups.

4.1. Amplitude recursions for geometries defined through power functions. Non-planar geometries defined through power functions have attracted attention in the literature, particularly in relation to exact solutions of shallow water equations [8].

When both $\bar{h}$ and $B$ are simple power functions it may be assumed that asymptotic expansions do exist with amplitudes as simple power functions as well.

$$
\bar{h}=h_{0} x^{\alpha}, \quad B=B_{0} x^{\beta}, \quad A_{j}=C_{j} x^{q_{j}} .
$$

Insertion of these expressions in (3.6), with $x_{r}$ as 0 or $\infty$ according to the power in the integral, provides us with recursion formulas for $C_{j}$ and $q_{j}$. The different choices of $x_{r}$ are required to obtain $A_{j}$ as a single power, but will lead to a jump in the solution when $\alpha$ crosses 2 . For $\alpha \neq 2$, we may write

$$
q_{j}=-p-\mu j, \quad C_{j+1}=\nu_{j} b_{j} C_{j},
$$

where $p=\frac{1}{4} \alpha+\frac{1}{2} \beta, \mu=1-\frac{1}{2} \alpha$ and

$$
\nu_{j}=\frac{1}{2} h_{0}^{\frac{1}{2}} \mu(j+1), \quad b_{j}=\left(1-\frac{\mu-p}{(j+1) \mu}\right)\left(1-\frac{p}{(j+1) \mu}\right) .
$$

For $j \rightarrow \infty$ the factor $b_{j}$ approaches unity, whereas the factor $\nu_{j}$ causes $C_{j}$ to grow fast with $j$. However, the discussion of the implications for the series expansion for $\eta$ awaits specification of the shape functions $F_{j}$.

Expressions can be made more compact by introducing $\tau(x)=h_{0}^{-\frac{1}{2}} \mu^{-1} x^{\mu}$, which is the travel time from 0 to $x$ if $\alpha<2$, and the travel time from $\infty$ to $x$ if $\alpha>2$. For waves 
propagating toward decreasing $x$ the amplitudes $A_{j}$ and the phase function, defined in (3.2), may be written

$$
A_{j}=j !(2 \tau(x))^{-j}\left(\prod_{i=1}^{j} b_{j}\right) A_{0}, \quad \Theta=\kappa\left(\tau(x)-\tau\left(x_{0}\right)+t\right),
$$

where $x_{0}$ is the reference position for which $\Theta=0$ at $t=0$.

A change in the properties of the asymptotic expansion occurs at $\alpha=2$. For smaller values of $\alpha$ the shoreline, $x=0$, is reached in a finite time and the $A_{j}$ are expressed as inverse powers of $x$. For $\alpha>2$ the shoreline is not reached (in linear theory) and the $A_{j}$ inherit positive powers of $x$ for sufficiently large $j$. For $\alpha=2$ an alternative recursion, involving logarithmic terms, can be devised. This, and a discussion of solutions for $\alpha$ values close to 2, are found in section SM2.

4.2. Exact solutions. The most apparent example of a ray solution that becomes exact is $B \bar{h}^{\frac{1}{2}}=$ const. Then $A_{0}$ in (3.4) is constant, such that the series (3.3) and (3.7) truncate after the first term, and $\eta=A_{0} F_{0}, u=-\bar{h}^{-\frac{1}{2}} A_{0} F_{0}$ form an exact solution. Correspondingly, when the right hand side of the amplitude recursion in (3.5) vanishes for $j=0$ the amplitudes $A_{1}$, $A_{2}$ etc. become zero and $\eta=A_{0} F_{0}$ is still an exact solution, even though $A_{0}$ is non-constant. A more general group of exact solutions is found when $A_{1}$ is required to be constant, but not necessarily zero. Then, $A_{2}$ etc, becomes zero and $\eta=A_{0} F_{0}+\kappa^{-1} A_{1} F_{1}$ is an exact solution. For constant $A_{1}$ and $j=0$ the rightmost relation in (3.5) is integrated to give

$$
A_{0, x}=-\left(A_{1} \bar{h}^{-\frac{1}{2}}+D \sigma^{2}(B \bar{h})^{-1}\right)=-\bar{h}^{-\frac{1}{2}}\left(A_{1}+D A_{0}^{2}\right),
$$

where $D$ is a arbitrary constant and (3.4) has been invoked. For $A_{1}=0$ equation (4.4) yields

$$
A_{0}(C+D \tau(x))=1 \quad \text { or, equivalently } \quad B=\bar{h}^{-\frac{1}{2}}\left(C+D \int \bar{h}^{-\frac{1}{2}} d x\right)^{2},
$$

where $C$ is another constant. For $D=0$ the case $B \bar{h}^{\frac{1}{2}}=$ const. is reproduced, while $\bar{h}=$ $h_{0}=$ const. yields quadratic width $B=\left(C+D h_{0}^{-\frac{1}{2}} x\right)^{2}$. For channels of constant width (4.5) is readily integrated again to $\bar{h}=h_{0}(x+\text { const. })^{\frac{4}{3}}$, which is discussed in [33, 10].

When $A_{1}=0$ the surface elevation is completely described by the principal wave and $\eta$ approaches zero behind it, provided $F_{0}$ approaches, or becomes, zero for large arguments. Still, combining (4.4) with (3.7) we find $u=-\bar{h}^{-\frac{1}{2}} A_{0} F_{0}-D \sigma^{2}(\kappa \bar{h} B)^{-1} F_{1}$ which implies a trailing system with a volume flux of constant strength $q=D \sigma^{2} F_{1}(\infty) / \kappa$. The lack of a surface elevation in the trailing system has been interpreted as lack of reflection. Through comparison with solutions for a uniform channel we may well construe another interpretation. In a uniform channel the solution may be written $\eta=A_{0} F\left(t+\bar{h}^{-\frac{1}{2}} x\right)+B_{0} G\left(t-\bar{h}^{-\frac{1}{2}} x\right)$, $u=-\bar{h}^{\frac{1}{2}} A_{0} F\left(t+\bar{h}^{-\frac{1}{2}} x\right)+\bar{h}^{\frac{1}{2}} B_{0} G\left(t-\bar{h}^{-\frac{1}{2}} x\right)$, where $\bar{h}, A_{0}$ and $B_{0}$ now are constant. A volume flux of constant strength $q$ may then be obtained by choosing $G=F=$ const. and $-A_{0}=B_{0}=\frac{1}{2} q /\left(\bar{h}^{\frac{1}{2}} F\right)$. In view of this we may describe the trailing system as combination two progressive waves; one following the principal wave and one being a reflection. In a 
nonuniform channel this argument is debateable, not least since there is no obvious definition of pure unidirectional wave. Be that as it may, it is more clearly important that the presence of the trailing system leads to an irreversible energy loss in the principal wave (more details in section SM7). Moreover, as will be demonstrated in section 5.2.2, the trailing volume flux will couple to reflections from an apex combining an uniform and a non-uniform channel section.

For nonzero $A_{1}$ the equation (4.4) integrates to expressions

$$
A_{0}=C-A_{1} \tau, A_{0}=K^{-1} \tan \left(C-A_{1} K \tau\right), A_{0}=K^{-1} \tanh \left(C-A_{1} K \tau\right), K=\sqrt{\left|\frac{D}{A_{1}}\right|},
$$

for $D=0, D / A_{1}>0$ and $D / A_{1}<0$, respectively. The above relations may readily give $B$ expressed in terms of $\bar{h}$. If $F_{0}$ has compact support there is a constant surface elevation in the trailing system. When $D=0$ (3.7) and (4.4) imply $u=-\bar{h}^{-\frac{1}{2}} A_{0} F_{0}$. The water is then quiescent in the trailing system. On the other hand, there is volume flux of constant strength there if $D$ is nonzero. When the width is constant the leftmost relation in (4.6) gives $h=h_{0}(x+\text { const. })^{4}$.

4.2.1. Exact solutions for geometries defined by power functions. [8] studied channels where the geometries were defined by power functions and discovered cases where the surface elevations from the basic ray solution (3.1) were exact. Below we will show that these are only the first in a sequence of exact solutions of increasing complexity. (4.2) and (3.5) imply that the asymptotic series will truncate after term $n\left(C_{n+1}=0\right)$, provided $b_{n}=0$. This gives two families of exact solutions,

$$
\alpha_{n}^{(i)}=\frac{4 n+4-2 \beta}{2 n+3}, \quad \alpha_{n}^{(i i)}=\frac{4 n+2 \beta}{2 n-1} .
$$

In both cases $\alpha_{n}$ approaches 2 as $n \rightarrow \infty$. Moreover, $\alpha_{n}^{(i)}<2$ and $\alpha_{n}^{(i i)}>2$ for all $n$. Naturally, a truncated series means that the remaining finite sum is an exact solution.

For the first family we have cancellation after a single term in (3.3) when $\alpha=\frac{4}{3}-\frac{2}{3} \beta$. For $\beta=0$ the case $\alpha=\frac{4}{3}$ is reproduced. Another interesting case is $\alpha=1, \beta=\frac{1}{2}$ which may correspond to an inclined channel with parabolic cross-sections [5]. The series (3.7) for $u$ will contain two terms in these cases. For $\beta=0$ we find $\alpha_{1}^{(i)}=\frac{8}{5}$. Then the exact expressions for $\eta$ and $u$ have two and three terms, respectively. For larger $n$ the solutions may contain strong modifications of the principal wave (examples will be given in sec. 4.4).

For the second group there is cancellation after $n=0$ only for the trivial case $\bar{h}^{-\frac{1}{4}} B^{-\frac{1}{2}}=$ const. For $\beta=0$ we find $\alpha_{1}^{(i i)}=4$. While (3.3) now yields a two term expression for $\eta$, only a single term remains for $u$. For all $\alpha_{n}^{(i i)}$ the factor $\bar{h}^{-\frac{1}{2}} A_{n}+A_{n-1, x}$ in (3.7) vanishes, making the series for $u$ one term shorter than that of $\eta$. For $n=1$ and an $F_{0}$ that either has compact support, or is tapering off exponentially at the outskirts, the surface elevation approaches a constant for large $\Theta$.

It is stressed that the truncation of the series (3.3) does not imply that these solutions are of a different nature from those with a full series. In particular, energy is not better preserved in the principal wave, as shown in section SM7.1. Moreover, the variation of the wave characteristics with $\alpha$ and $\beta$ is continuous. 

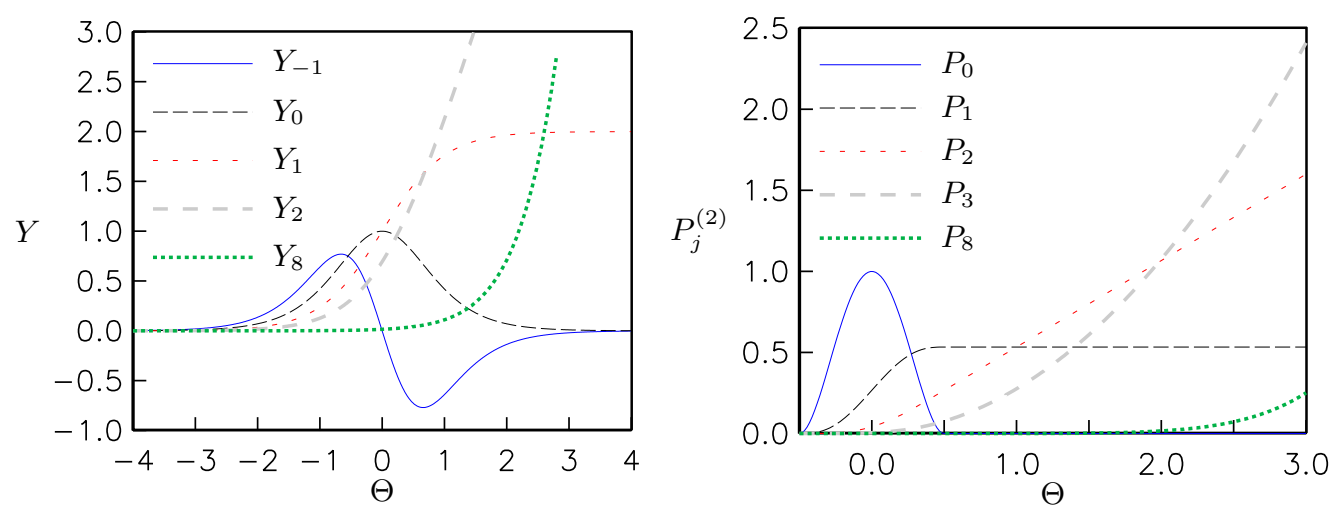

Figure 2. The shape functions. Left panel: hyperbolic function shapes. Right panel: piece-wise polynomials for $M=2$. Do observe the different extent (in $\Theta$ ) of the functions in the panels.

Exact solutions, similar to those described above, are described in section SM6.2 for geometries defined through exponentials.

4.3. Shape functions for the surface elevation. As mentioned in section 3.2 our expansion (3.3) becomes equivalent to the WKB method when, instead of a pulse, we invoke a periodic shape $F_{j}=(-i)^{j} \exp (i \Theta)$. Then $\left|A_{j} F_{j}\right|$ behaves like const. $\times\left(\sqrt{h_{0}}|\mu| \kappa^{-1}\right)^{j} j$ !. This gives the typical asymptotic and divergent series generally associated with WKB expansions.

Below two different shapes are investigated. Firstly, $F_{0}$ is given by hyperbolic functions that vanish exponentially at either side. Secondly, $F_{0}$ are defined by piece-wise polynomials and has compact support. The two options give series with quite different properties. Also other shapes have been used, but these gave little additional insight and are omitted.

4.3.1. The $\operatorname{sech}^{2}$ shape. A natural choice for the shape of the principal wave is $F_{0}(\Theta)=$ $a Y_{0}(\Theta)$ (single crest) or $F_{0}(\Theta)=a Y_{-1}(\Theta)$ (so called N-wave, [31]), where $a$ is an amplitude and $Y_{j}$ are hyperbolic functions defined as

$$
\begin{array}{ll}
Y_{-1}(\Theta)=-2 \cosh ^{-2} \Theta \tanh \Theta, & Y_{0}(\Theta)=\cosh ^{-2} \Theta \\
Y_{1}(\Theta)=\tanh (\Theta)+1, & Y_{2}(\Theta)=\log (2 \cosh (\Theta))+\Theta
\end{array}
$$

where each is the integral of the former. The subsequent functions, $Y_{3}$ etc., cannot be expressed that simply, but may be obtained by numerical integration (see appendix SM5.2 for details). The option $F_{0}=a Y_{0}$ implies $F_{j}=a Y_{j}$, while $F_{0}=a Y_{-1}$ leads to $F_{j}=a Y_{j-1}$. In the latter case a trailing system will appear first to order $\kappa^{-2}$ in (3.3). In constant depth $a Y_{0}$ is the leading order approximation to a solitary wave when $\beta=0$ and $\kappa=\sqrt{\frac{3 a}{4 h^{3}}} \frac{x_{c}}{h_{c}}$. However, we are working with linear shallow water equations and no relation between $\kappa$ and the wave height needs to be imposed. Shapes are depicted in figure 2. For $\Theta<0$ the $Y_{j}$ may be expanded 

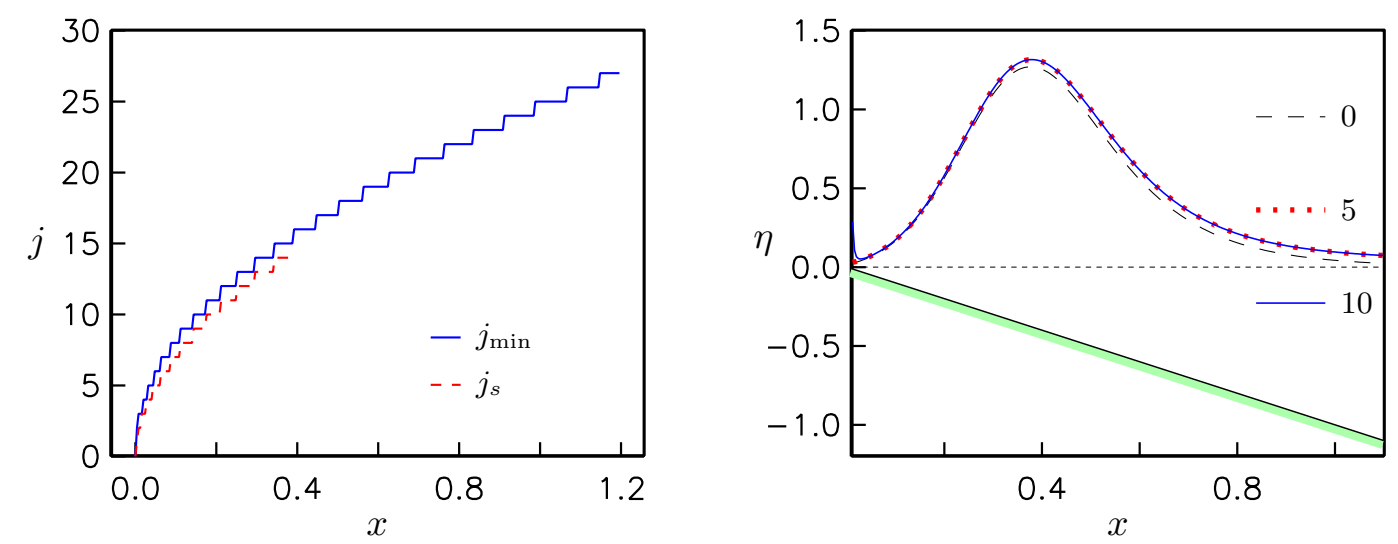

Figure 3. Properties of the asymptotic series for $\kappa=3, h_{0}=1, \alpha=1, x_{0}=1$ and $t=0.75$. Left panel: Number of minimum term, as defined in the text. Right panel: partial sums for $\eta$ with different number of terms. When 10 terms are retained the divergence is becoming visible near $x=0$.

according to

$$
Y_{j}=2^{2-j} \sum_{n=1}^{\infty}(-1)^{j+1} n^{1-j} e^{2 n \Theta}, \quad j \geq 0 .
$$

For $\Theta \ll 1$ we have $\left|Y_{j+1} / Y_{j}\right| \approx \frac{1}{2}$ and the series for $\eta$ again diverges since $A_{j}$ grows as $j$ ! (see (4.3)). The smallest term in the series (3.3) for $\Theta \ll 1$ is then found for $j_{s} \sim 4 \kappa|\tau(x)|=$ $4 \kappa x^{\mu} /\left(\sqrt{h_{0}}|\mu|\right)$. For illustration the case $\kappa=3, h_{0}=1, \alpha=1, \beta=0$, and $x_{0}=1$ is presented in figure 3 for the time when the wave is about to run up on the beach. The number of the minimum term, $j_{\min }$, increases with $x$ in the front of the wave, as indicated by the formula for $j_{s}$. Moreover, $j_{\min }$ continues to increase at the rear of the principal wave and in the tail. Depth profiles with $\alpha>2$ yields a $j_{\text {min }}$ that decreases with $x$ (results not shown). The different behaviours of $j_{\min }$ for $\alpha<2$ and $\alpha>2$ comply with the discussion in section SM2.1.

4.3.2. Piece-wise polynomial shape functions. An interval $J$, corresponding to $\Theta_{0} \leq$ $\Theta \leq \Theta_{L}$, is divided in $L$ sub-intervals. In each sub-interval $F_{0}$ may be represented as a polynomial in $\Theta$. This will define a principal wave which is truly confined to $J$, and which may inherit continuous derivatives of any order provided the local polynomials are chosen accordingly. Moreover, the integration to obtain $F_{j}$ is straightforward, although the expressions may become lengthy. Naturally, $F_{j}$, from some positive $j$ and on, will be nonzero for $\Theta>\Theta_{L}$.

In appendix $\mathrm{A}$ it is shown that if $F_{0}$ is any piece-wise polynomial with compact support, and $A_{j}$ is given through (4.2), the series (3.3) converges if (still $\mu=1-\frac{1}{2} \alpha$ )

$$
K \equiv \frac{\Theta-\Theta_{0}}{2 \kappa|\tau(x)|}=\frac{|\mu|}{2 \kappa} h_{0}^{\frac{1}{2}} x^{-\mu}\left(\Theta-\Theta_{0}\right)<1,
$$

and diverges if $K>1$. 
When the wave approaches the shore $(x=0)$ the front of the wave, $x_{f}(t)$ is given by $\Theta\left(x_{f}, t\right)=\Theta_{0}$. By means of (4.3) $\Theta-\Theta_{0}$ is then expressed by $x$ and $x_{f}$ and (4.10) is rewritten as

$$
K=\frac{1}{2}\left|1-\frac{\tau\left(x_{f}\right)}{\tau(x)}\right|=\frac{1}{2}\left|1-\left(\frac{x_{f}}{x}\right)^{\mu}\right|<1 .
$$

For $\alpha<2$ the series (3.3) thus converges everywhere until the wave front reaches the shoreline. Stopping the summation of the series after $A_{n} F_{n}$ we find a residue $-\kappa^{-n} B^{-1}\left(B \bar{h} A_{n, x}\right)_{x} F_{n}$ which goes to zero as $n \rightarrow \infty$. Provided that the wave equation on the form (2.2) is well posed, in the sense that vanishing residuals imply vanishing errors (demonstrated for the $\mathrm{L}_{2}$ norm in section SM8), the converged series is then an exact and complete solution. It is also noteworthy that the convergence is independent of $\kappa$, meaning that the solutions are valid regardless of wave length and hence go beyond what is normally conceived as ray theory. Since all continuous functions with compact support may be approximated to arbitrary accuracy by piece-wise polynomials, it follows that these properties apply to all $F_{0}$ with compact support. The above results are obtained with the amplitude recursion formula (3.6) for which wave propagation to the left, interpreted as $\Theta=\kappa(\tau+t)$, was assumed. However, this interpretation is strictly valid only in the limit $\kappa \rightarrow \infty$. For finite $\kappa$ the variables $x$ and $\Theta$ in the convergent series (3.3) are intrinsically mixed and the sum is $\eta(x, \Theta)$, which is equivalent to $\eta$ being a function of $x$ and $t$. Hence, (3.3) may contain a reflected wave. What may be seen as surprising is the fact that the possible reflected wave is inherent in an expansion of geometrical optics like (3.3). On the other hand, the notion of no reflection can be questioned even for the exact, closed form solutions in 4.2 .

The front of the incident wave reaches the shore at time $t_{c}=\tau\left(x_{0}\right)+\Theta_{0} / \kappa$. For larger times the front of the wave that is reflected from the shore is at $x_{R}$, where $\tau\left(x_{R}\right)=t-t_{c}$. When $\Theta-\Theta_{0}$ is expressed in terms of $x$ and $x_{R}$ the criterion (4.10) becomes

$$
K=\frac{1}{2}+\frac{1}{2} \frac{\tau\left(x_{R}\right)}{\tau(x)}=\frac{1}{2}+\frac{1}{2}\left(\frac{x_{R}}{x}\right)^{\mu}<1 .
$$

Hence, the series (3.3) will diverge in the region, adjacent to the shoreline, which is affected by a reflection. Outside this region, the series still converges.

For $\alpha>2$ equation (4.11) implies that (3.3) converges when $x<3^{\frac{2}{\alpha-2}} x_{f}$. This region decreases with $\alpha$ and diminishes as the wave approaches the shoreline.

In some subsequent examples $F_{0}(\Theta)=P_{0}^{(M)}$ will be used, where $P_{0}^{(M)}$ is a simple, normalized, piece-wise polynomial, with continuous derivatives through order $M-1$ :

$$
P_{0}^{(M)}=4^{M}\left(\Theta-\frac{1}{2}\right)^{M}\left(\Theta+\frac{1}{2}\right)^{M} \quad \text { for } \quad-\frac{1}{2}<\Theta<\frac{1}{2},
$$

and $P_{0}^{(M)}=0$ elsewhere. The integrals $F_{j}=P_{j}^{(M)}$ are given by (A.3) combined with (A.8). A selection of $P_{j}^{(M)}$ is depicted in figure 2 .

4.4. Evolution of wave on a slope; examples. The partial sum of (3.3) where the terms up to, and including, $O(\kappa)^{-n}$ are retained is now denoted by $\eta_{n}$. In a boundary value problem 
on the interval $x_{a} \leq x \leq x_{b}$ the approximation $\eta_{n}$ may then be used for initial conditions at $t=t_{0}$, as well as boundary conditions at $x=x_{a}$ and $x=x_{b}$. The computed solution is denoted as $\eta_{n \text { :numerical }}$. Then, the temporal growth of the error $\Delta \eta_{n}=\eta_{n \text { :numerical }}-\eta_{n}$ will give an indication of the accuracy of $\eta_{n}$. Naturally, this requires that the discretization error of the numerical solution is much less than $\eta-\eta_{n}$. The geometry is normalized as to give $h_{0}=1$ and $x_{0}=1$. The initial time, $t_{0}$, may be chosen.

For the inclined plane $(\alpha=1, \beta=0)$ the first few terms of (3.3) read

$$
\frac{\eta}{C_{0}} \sim x^{-\frac{1}{4}}\left(F_{0}(\Theta)+\frac{1}{16 \kappa x^{\frac{1}{2}}} F_{1}(\Theta)+\frac{9}{512 \kappa^{2} x} F_{2}(\Theta)+\frac{147}{8192 \kappa^{3} x^{\frac{3}{2}}} F_{3}(\Theta)+\ldots\right),
$$

where $\Theta=\kappa(2 \sqrt{x}-2+t)$ and errors of order $\kappa^{-4}$ are implicit.

For $n=0$ and $\kappa=4$ figure 4 shows errors up to $2.3 \%$ at a time when the wave has reached the left boundary $x_{a}=0.05$. In fact, the trailing system, which appears in the higher orders in (3.3), gives an elevation that is larger than the errors observed. However, the trailing system is modified when $\eta_{0}$ is used for the boundary condition (more details in section SM3.3). For $n=6$ and $x>0.05$, we have errors as small as $\left|\Delta \eta_{6}\right|<2.2 \cdot 10^{-5}$. The norm $\mathrm{L}_{2}=\left\{\left(x_{b}-x_{a}\right)^{-1} \int_{x_{a}}^{x_{b}}(\Delta \eta)^{2} d x\right\}^{\frac{1}{2}}$ of $\Delta \eta_{6}$ is $1.5 \cdot 10^{-5}$, while the corresponding theoretical $\mathrm{L}_{2}$ bound from section SM8 is $4 \cdot 10^{-4}$. Optimization of the number of terms, in the sense of stopping the summation at smallest one, reduces these errors only slightly. More examples are given in section SM2.5.

As shown in figure 5 the trailing systems of the exact solutions, with $\alpha$ given by (4.7), become increasingly strong with increasing $n$ (number of terms in exact solution). Also the heights of the leading pulses are strongly modified.

The expansion provides accurate, or sometimes even exact, mathematical solutions also for cases with $\alpha$ close to and above 2. However, the results look strange and have strayed far from the principal wave shape. When an $\alpha$ near 2 is employed in the geometry with an apex (section 5) none of the strange features appear due to a modification of the wave shape (section SM3.2).

5. Waves conveyed to a non-uniform channel. The simple power function solutions described in section 4.1 display some puzzling properties. First, no reflected wave is clearly discernible in the solutions. Then, as shown in figure 5, the substantial changes of the wave shapes due to the higher order terms in the expansions become visible. However, the wave forms, such as (4.14), are merely mathematical solutions on a special class of slopes, as the waves are presented without an origin or a generation mechanism. To put the expansions into perspective propagation of waves from uniform channel section into a non-uniform one, through a apex, is described. The expansion (3.3) is employed in the variable part, while the solution in the uniform region is described by waves of permanent form. Then the local solutions are patched at the apex by requirements of continuity. Relevant examples from the literature, where such patching is used, are $[30,12,11,23]$. Two problems related to that of transmission at the apex are outlined in section SM3.

5.1. Transmission at an apex. An example sketch of a channel transect is shown in figure 6. For $x>x_{0}$ both $B$ and $\bar{h}$ are constant, while the channel is non-uniform for $x<x_{0}$. At $x_{0}$ 

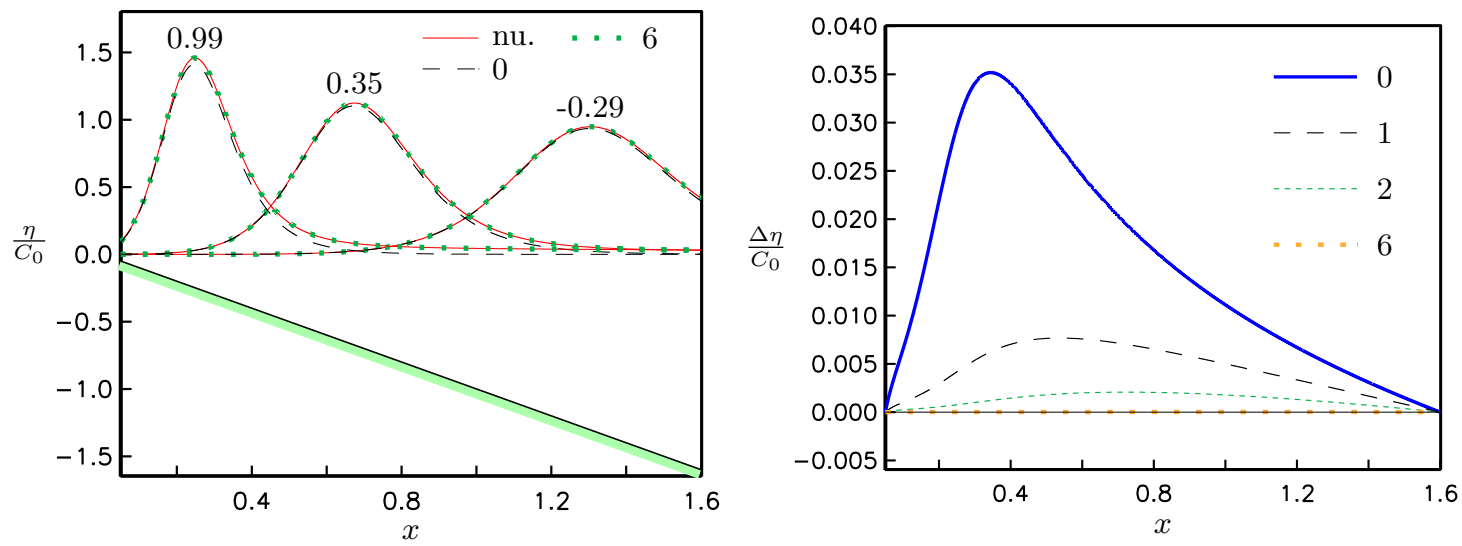

Figure 4. Normalized surfaces on an inclined plane for $F_{0}=Y_{0}, \kappa=4, x_{a}=0.05, x_{b}=1.6$ and $t_{0}=-1.57$. Left panel: numerical surface elevation and selected $\eta_{n}$ at times as given above the crests. Right panel: $\Delta \eta_{n}$, marked by $n$, as explained in the text, for the last time displayed in the left panel. The thin solid line marks zero.
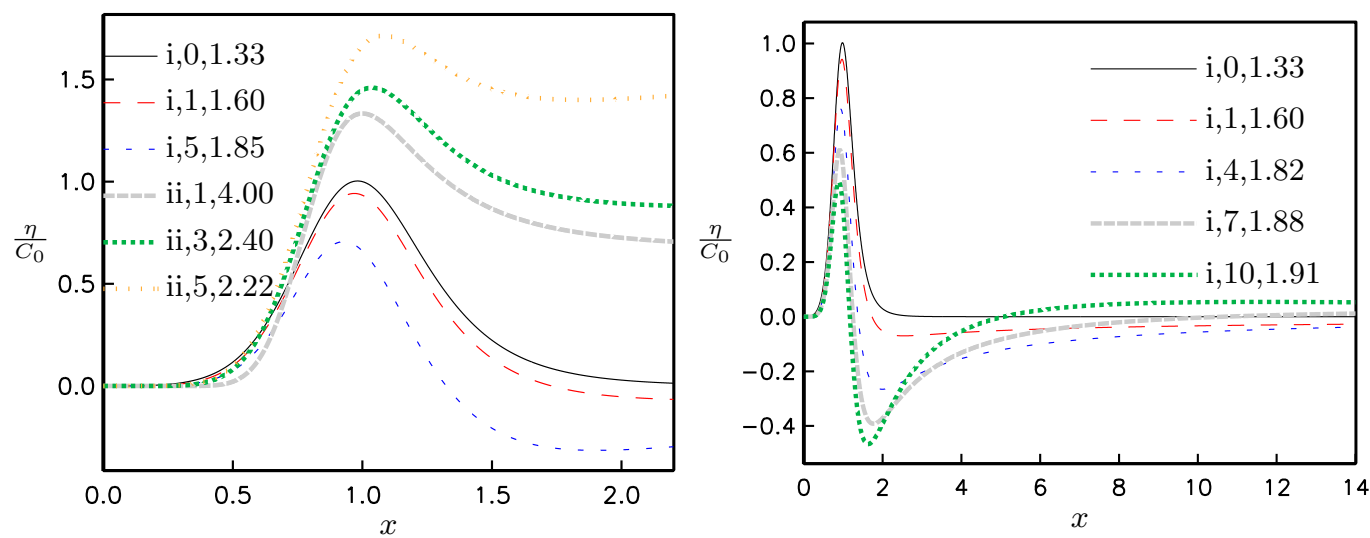

Figure 5. Surfaces from selected exact solutions for $F_{0}=Y_{0}, \kappa=3, \beta=0$ and $t_{0}=0$. Curves are marked by family, value of $n$ and value of $\alpha$ according to (4.7).

we have continuous $B$ and $\bar{h}$, but there may be jumps in their derivatives. Still, $x_{0}$ is also the location where $\Theta=0$ at $t=0$, as appearing in (3.2). Moreover, it is also crucial to choose $x_{r}$ in (3.6) equal to $x_{0}$. Scales are chosen as to give $B=\bar{h}=1$ for $x>x_{0}$.

At $x_{0}$ the fluxes of mass, momentum and energy must be continuous, which gives continuous $\eta$ and $u$ (see, for instance, section SM7). The surface elevation of the incident wave, $I$, is specified while the reflected wave, represented by the elevation $R$, and the fields of the transmitted wave, denoted by $\eta^{(t)}$ and $u^{(t)}$, must be found. For the transmitted wave the shape $\left(F_{0}\right)$ and the amplitude $\left(A_{0}\left(x_{0}\right)\right)$ of the principal wave must be determined. The rest 


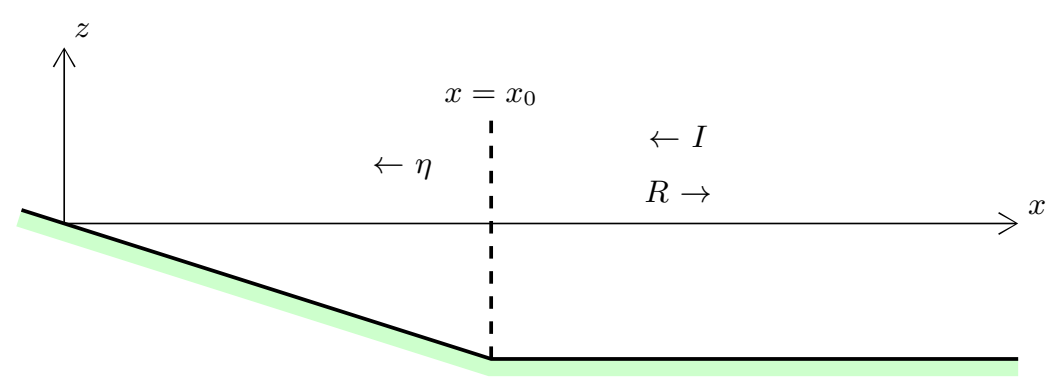

Figure 6. Sketch of geometry inspired by wave tanks.

of (3.3) and (3.7) then follows from (3.4), and the recursion formulas (3.5) and (3.6).

For $x>x_{0}$ it is convenient to write the solution according to

$$
\eta=I+R, I=I\left(\Theta_{i}\right), \quad R=R\left(\Theta_{r}\right), \Theta_{i}=\kappa\left(t+\left(x-x_{0}\right)\right), \quad \Theta_{r}=\kappa\left(t-\left(x-x_{0}\right)\right) .
$$

At the apex all the phases then become equal $\Theta_{i}=\Theta_{r}=\Theta=\kappa t$. Continuity of $\eta$ and $u$, respectively, yield the following relation at $x=x_{0}$

$$
\left\{\begin{array}{ccc}
\sum_{i=0}^{(t)}\left(x_{0}, t\right) & \sim \kappa^{-i} A_{i} F_{i}(\Theta) & \sim I(\Theta)+R(\Theta), \\
u^{(t)}\left(x_{0}, t\right) & \sim-\left(\sum_{i=0}^{\infty} \kappa^{-i} A_{i} F_{i}(\Theta)+\sum_{i=1}^{\infty} \kappa^{-i} A_{i-1, x} F_{i}(\Theta)\right) & \sim-I(\Theta)+R(\Theta),
\end{array}\right.
$$

Combining the two we find

$$
I(\Theta) \sim A_{0} F_{0}(\Theta)+\sum_{i=1}^{\infty} \kappa^{-i}\left(A_{i}+\frac{1}{2} A_{i-1, x}\right) F_{i}(\Theta) .
$$

Since (3.6), with $x_{r}=x_{0}$, yields $A_{j+1}\left(x_{0}\right)=-\frac{1}{2} A_{j, x}\left(x_{0}\right)$ the sum vanishes and we obtain

$$
A_{0}\left(x_{0}\right) F_{0}=I, \quad R(\Theta) \sim-\frac{1}{2} \sum_{i=1}^{\infty} \kappa^{-i} A_{i-1, x}\left(x_{0}\right) F_{i}(\Theta)=O\left(\kappa^{-1}\right) .
$$

When the integral of the incident wave, $V=\int_{-\infty}^{\infty} I d \Theta=A_{0}\left(x_{0}\right) F_{1}(\infty)$, is non-zero the leading order reflection will be a surface elevation of height $V H_{r}$, where $H_{r}=\kappa^{-1}\left(\frac{1}{8} \frac{d \bar{h}}{d x}+\frac{1}{4} \frac{d B}{d x}\right)$. This is a long wave that persists after the passing of the incident wave. The incident shape $I=a Y_{-1}$ yields $V=0$ and the leading surface reflection is $a H_{r} Y_{0}$. If the transition at $x_{0}$ is smooth, in the sense that the first derivatives of $B$ and $\bar{h}$ are zero, the leading order reflected wave becomes $-A_{0}\left(x_{0}\right) \kappa^{-2}\left(\frac{1}{16} \frac{d^{2} \bar{h}}{d x^{2}}+\frac{1}{8} \frac{d^{2} B}{d x^{2}}\right) F_{2}$.

Provided $V>0$ and that the wave is amplifying $\left(A_{0, x}<0\right)$ for $x<x_{0}$ the equation (3.6) yields $A_{1}\left(x_{0}\right)>0$. This implies that the wave height is increased in the transmission, which 

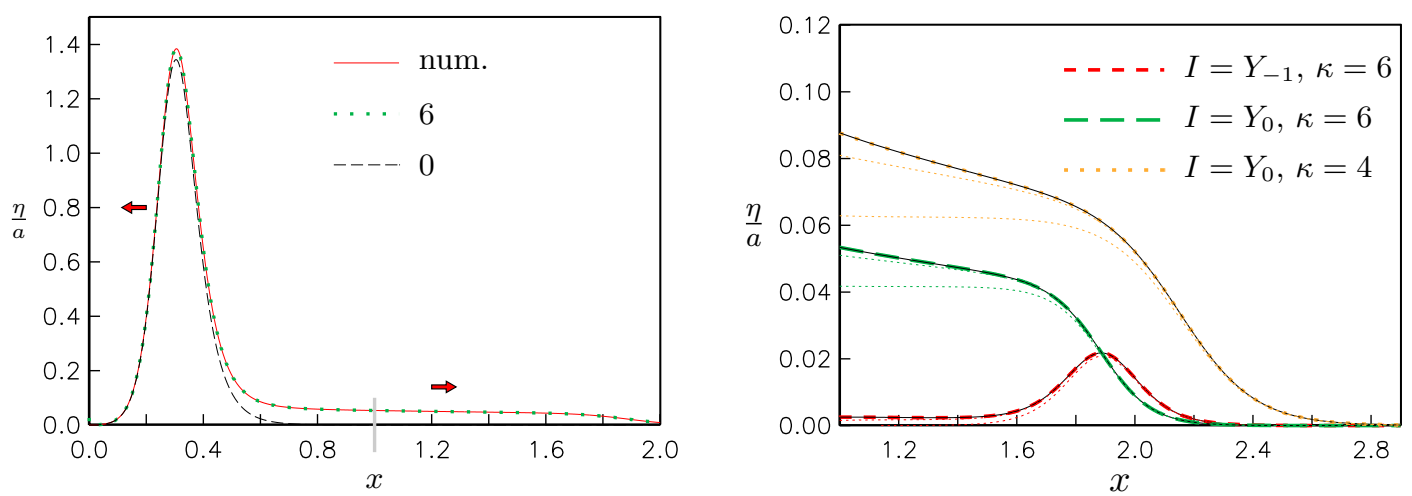

Figure 7. Reflection at an apex. Left panel: Normalized surfaces for $F_{0}=I=a Y_{0}, \alpha=1, \beta=0$ and $\kappa=6$. Right panel: The reflected waves for a few selected cases. The bold dashed curves in the legend correspond to $\eta_{6}$, numerical solutions are depicted with thin solid lines and $\eta_{1}, \eta_{2}$ with thin dashes. The latter two may be distinguished by $\eta_{1}$ approaching a constant as $x \rightarrow 1^{+}$

may seem counter intuitive as the energy of the incident wave must support both transmission and reflection. However, this is resolved through the modified energy densities on the slope as elaborated in SM7.4.

\subsection{Examples of transmitted waves.}

5.2.1. Transmission to an inclined plane. For constant channel width and $\bar{h}=x$ we have $x_{0}=1$ and the amplitude recursion yields

$$
\eta \sim x^{-\frac{1}{4}} F_{0}+\frac{1}{16 \kappa}\left(x^{-\frac{3}{4}}+x^{-\frac{1}{4}}\right) F_{1}+\frac{1}{512 \kappa^{2}}\left(9 x^{-\frac{5}{4}}+2 x^{-\frac{3}{4}}+5 x^{-\frac{1}{4}}\right) F_{2}+O\left(\kappa^{-3}\right),
$$

where $F_{0}=I$ and the phase is $\Theta=\kappa(2 \sqrt{x}-2+t)$. Comparing (5.5) with (4.14) we observe an extra term in $A_{1}$ with the same $x$-dependence as $A_{0}$ (const. $\left.\times x^{-\frac{1}{4}}\right)$. This is a modification of the wave shape which, in turn, leads to the mid term in $A_{2}$. The third term in $A_{2}$ is a shape modification again, and in this manner the series expansion continues. The reflected wave becomes

$$
R=\frac{\alpha}{8 \kappa} F_{1}\left(\Theta_{r}\right)+\frac{\alpha^{2}}{32 \kappa^{2}} F_{2}\left(\Theta_{r}\right)+O\left(\kappa^{-3}\right) .
$$

Relating the coefficient of (5.6) and (5.5) in view of the continuity for $\eta$, we observe that half of the leading order reflection (the $F_{1}$ term) is linked to the shape change in the transmission, while the other half is linked to the higher order terms in the expansion on the slope.

Now the shape of the incident wave is chosen as either $I=a Y_{0}$, or $I=a Y_{-1}$ where $a$ is an amplitude factor and $Y_{j}$ is as defined in (4.8). Figure 7, right panel, shows that the asymptotic expansion reproduces the qualitative features of the transmission with a few terms retained. On the other hand, for the seven term approximation $\left(\eta_{6}\right)$ the error is of order $10^{-5}$ in finite depth $\left(x>0.05\right.$, say) for the time shown. In the reflection from the $\mathrm{N}$-wave $\left(I=a Y_{-1}\right)$ there is a long $O\left(\kappa^{-2}\right)$ tail in addition to the single $\left(\kappa^{-1}\right)$ pulse, with shape $Y_{0}$. 
The modified shape due to the transmission will eventually affect the runup of the shoreline (see [23] for the case with a parabolic channel). For instance, the absence of the shape change leads to a mild undershoot of maximum runup height by the celebrated runup formula by Synolakis [30]. Details become a bit involved and are left for SM4.

5.2.2. Transmission when closed form solutions do exist. A naive presumption is that an incident pulse, $\eta=I$, is completely transmitted to $\eta=A_{0} I(\Theta)$ when this is an exact solution. However, such a solution is not feasible since the principal wave has a trailing system with a stationary fluid flow in this case, as discussed in section 4.2. This flow is directed toward the apex and the volume transport implies a reflected wave propagating from the apex and into the uniform channel section. Continuous surface elevation then again requires a modified shape in the transmitted surface in the form of a long tail. The latter is evident when the amplitude recursion (3.6) is used with $x_{r}=x_{0}$ and $\alpha=\alpha_{0}^{(i)}$ (from (4.7))

$$
\eta \sim A_{0}\left(x_{0}\right) x^{-\frac{\beta+1}{3}} \sum_{j=0}^{\infty}\left(\frac{\beta+1}{6 \kappa}\right)^{j} F_{j}(\Theta) .
$$

Here the series part only modifies the shape to produce a tail. Correspondingly there is a reflected long wave. Interestingly, in their study of the $\alpha=\frac{4}{3}, \beta=0$ case [12] obtained an exponential decay in $\eta$ at the apex when the wave propagated from a flat, shallow shelf into a deeper region defined by the curved depth profile. In our case (wave amplified after the transmission) there is a slow growth in $\eta\left(x_{0}, t\right)$, in line with what is observed for an inclined plane in figure 7. The properties of the transmission, as well as the relation between (5.7) and references, are elaborated in section SM3.2.2.

6. Concluding remarks. Application of geometrical optics provides formal asymptotic series for rather general wave shapes and geometries. The higher order terms yield a trailing structure with slowly varying surface elevations and velocities. Explicit expressions for the amplitudes are obtained for special geometries, such as those being defined by single power functions or exponentials and a few cases where the arithmetics are simplified by the use of the depth as the spatial coordinate (latter two types in supplement only). Both for the power functions and the exponentials families of exact solutions are discovered. Previous exact solutions from the literature are the most basic members of these families. However, no special properties concerning amplification or conservation of energy etc. are discovered for any of the exact solutions.

The asymptotic expansions for channels defined by power functions lend themselves to detailed analysis for some choices of surface shapes. When the principal shape is a squared hyperbolic secant function, akin to a solitary wave, the result is a divergent asymptotic series as might be expected. Still, even for only moderately short waves a large number of terms may be retained and close approximations may be obtained. On the other hand, for principal shapes defined by polynomials with compact support the series are convergent, regardless of wavelength and spatial position, as long as the power for the depth is less than 2 and the wave has not yet reached the shoreline. This is quite surprising in view of the starting point for the asymptotic expansion, namely that wave lengths are short in relation to the variation of the channel geometry. Moreover, also the reflected waves, if there are any, are then inherent 
in the geometrical optics. A similar behaviour is found when the power of the depth function is larger than 2, or if the the geometry is defined by exponentials (sec. SM6), but then the regions with convergence are defined differently. On the other hand, for the more complex depth profile in sec. SM3.4, where $\bar{h}$ is given by a hyperbolic tangent, a gradual reflection from a sloping bottom is identified. This reflection is then not a part of series from the geometrical optics, which presumably does not converge in this case even for principal shapes of compact support. Special properties of geometries given by power functions and exponentials, such as monotonic derivatives of any order, may readily be pointed out, but do not amount to an explanation of the different behaviour from, say, the depth profile defined by the hyperbolic tangent.

To put the asymptotic expansion into context the transmission of a pulse from a uniform channel section to a varying section defined through power functions is studied. In the sloping part of the channel a modified expansion is devised and comparison with numerical solutions shows that a high accuracy may be obtained. The transmission at the apex (discontinuous derivatives of the geometry) joining the sections is counter-intuitive in the sense that the wave height is increased. Still, the energy of the transmitted wave system is smaller than that of the incident wave, which leaves energy also for the reflection from the apex. Also the shape is changed in the transmission to give tails in the surface elevation and velocity field. This shape change is linked to the reflection from the apex and will also appear when simple analytic solutions exist in the non-uniform channel section. As a consequence, infinite series appear even in such cases. In general the tails generated in the transmission will combine with the trailing system inherent in the asymptotic expansions for the transmitted wave.

Kept within the framework of linear shallow water theory, the asymptotic expansion may presumably be generalized to simpler cases of non-planar waves and more general bathymetries. Evolution along ray lobes is a possibility, but explicit and simple solutions may be hard to obtain. Extensions to more general equations, with nonlinearity and dispersion, are more difficult. First, to retain the concept of well defined principal waves the effects of dispersion/non-linearity would either need to be very weak, such as for an asymptotic wave front for large times [19], or in a balance, such as for solitary waves. In either case one would likely need to include a slow time scale as well as the slow length scale. In the literature $[14,20,16,17,22]$ ray theory has been applied to solitary waves with a laterally non-uniform shape or in a bathymetry. However, the requirement on the variations of wave characteristics and bathymetry is then quite severe. The variation needs to be slow in relation not only to the wavelength, but also compared to nonlinear and dispersive scales [21]. This is quite contrary to what is found for the solutions which are presented herein, where the limitations of this kind are weak or even fully absent. For any of the possible extensions mentioned, the prospect of finding full series, let alone analyzing their properties, is meagre.

Appendix A. Convergence for polynomial shapes. A polynomial is defined, for all $\Theta$, as

$$
H_{0}=\sum_{n=N}^{M} a_{n}\left(\Theta-\Theta_{a}\right)^{n},
$$

where $0 \leq N \leq M$ and $a_{N} \neq 0$. A polynomial with compact support is defined as $P_{0}=H_{0}$ 
for $\Theta_{a} \leq \Theta \leq \Theta_{b}$ and $P_{0}=0$ elsewhere. Then, recursively, we define $P_{j}=\int_{-\infty}^{\Theta} P_{j-1}(\hat{\Theta}) d \hat{\Theta}$, for $j=1,2 \ldots$. Some degree of smoothness at the interval boundaries is required for $F_{0}=P_{0}$ if (3.3) is to yield a formally correct solution of (2.2). However, no such limitations are needed at this stage. Smoothness may later be obtained by combining several polynomials such as in the B-spline. Higher $P_{j}$ will anyhow be increasingly smooth. Through the definition of the polynomials (defined for all $\Theta$ )

$$
H_{j} \equiv \sum_{n=N}^{M} \frac{n !}{(n+j) !} a_{n}\left(\Theta-\Theta_{a}\right)^{n+j}, \quad T_{j} \equiv \sum_{n=0}^{j-1} \frac{H_{j-n}\left(\Theta_{b}\right)}{n !}\left(\Theta-\Theta_{b}\right)^{n}
$$

straightforward integration of (A.1) gives

$$
P_{j}= \begin{cases}0 & \text { for } \Theta<\Theta_{a}, \\ H_{j} & \text { for } \Theta_{a} \leq \Theta \leq \Theta_{b}, \\ T_{j} & \text { for } \Theta_{b}<\Theta .\end{cases}
$$

Since $H_{j-n}=\frac{d^{n} H_{j}}{d x^{n}}$ it is clear from the formula that $T_{j}$ is the Taylor polynomial of degree $j-1$ for $H_{j}$ around $\Theta=\Theta_{b}$. The presence of the $n$ ! and $(n+j)$ ! in the denominators of (A.2) changes the convergence properties in comparison to the $Y_{j}$ profiles. Rewriting $H_{j}$ we find

$$
\begin{aligned}
H_{j}=\frac{N !}{(N+j) !} a_{N}\left(\Theta-\Theta_{a}\right)^{N+j}\left(1+\frac{(N+1) a_{N+1}}{(N+j+1) a_{N}}\left(\Theta-\Theta_{a}\right)+\right. & \\
& \left.\quad \frac{(N+1) \cdots \cdot(N+M) a_{M}}{(N+j+1) \cdots \cdot(N+j+M) a_{N}}\left(\Theta-\Theta_{a}\right)^{M-N}\right) .
\end{aligned}
$$

When $j \rightarrow \infty$ the expression within rightmost parentheses approaches unity. Identifying $F_{j}=H_{j}$ and invoking (4.2) we find

$$
\lim _{j \rightarrow \infty}\left|\frac{A_{j+1} F_{j+1}}{A_{j} F_{j}}\right|=\frac{|\mu|}{2 \kappa} h_{0}^{\frac{1}{2}} x^{-\mu}\left(\Theta-\Theta_{a}\right)=K .
$$

Hence for $\Theta_{a} \leq \Theta \leq \Theta_{b}$ the ratio criterion yields that the series (3.3) converges if $K<1$ and diverges if $K>1$.

For $\Theta>\Theta_{b}$ we exploit that $T_{j}$ is the Taylor polynomial of $H_{j}$ to rewrite $T_{j}=H_{j}-E_{j}$. The Taylor residual term becomes $E_{j}=H_{0}\left(\Theta_{c}^{(j)}\right)\left(\Theta-\Theta_{a}\right)^{j} / j$ ! for some $\Theta_{c}^{(j)}$ between $\Theta_{b}$ and $\Theta$. Splitting the series (3.3) in two we may write $\left(\Theta>\Theta_{b}\right)$

$$
\eta \sim \sum_{j} A_{j} T_{j}=\sum_{j} A_{j} H_{j}-\sum_{j} A_{j} E_{j}
$$

The first series converges if $K$, still as defined in (A.5), is less than unity. The second series is dominated by another series according to

$$
\left|\sum_{j} A_{j} E_{j}\right| \leq \hat{H} \sum_{j}\left|A_{j}\right| \frac{\left(\Theta-\Theta_{b}\right)^{j}}{j !},
$$


where $\hat{H}$ is the maximum of $H_{0}$ between $\Theta_{b}$ and $\Theta$. Using the ratio criterion for the rightmost series in (A.7) we find a requirement for convergence which is weakened in relation to (A.5) by the replacement of $\Theta-\Theta_{a}$ by $\Theta-\Theta_{b}$. Hence, $K<1$ is a sufficient criterion for convergence for all $\Theta$ and $x$. When $K$ is just above 1 the first series in (A.6) diverges, while the second still converges. As a consequence the total series diverges. For larger $K$ both series diverge, but they diverge at different rates. Hence, cancellation may not produce convergence and we may state that the series for $\eta$ diverges for all $K>1$, also for $\Theta>\Theta_{b}$.

For a piece-wise polynomial an interval $\left[\Theta_{a}, \Theta_{b}\right]$ is subdivided into intervals through $\Theta_{a}=$ $\Theta_{0}<\Theta_{1}<. .<\Theta_{L}=\Theta_{b}$. The contributions from each sub-interval to $F_{j}$, and hence to the series (3.3) for $\eta$, may then be analyzed separately. For the polynomial belonging to the subinterval $i,\left[\Theta_{i-1}, \Theta_{i}\right]$, we then have the convergence criterion $K_{i}<1$, where $K_{i}$ is obtained by replacing $\Theta_{a}$ by $\Theta_{i-1}$ in (A.5). The strongest criterion then comes from the leftmost interval, which means that $K<1$ assures convergence for all intervals. Moreover, when $K>1$ the series for the first interval will diverge and it will also dominate the series from other intervals, even if they also diverge, since $K>K_{i}$ for $i>0$. Hence the total series for $\eta$ will diverge when $K>1$.

For $P_{0}=P_{0}^{(M)}$ the polynomials required in (A.3) become

$$
\left\{\begin{array}{l}
H_{j}=H_{j}^{(M)} \equiv 4^{M} \sum_{n=0}^{M}(-1)^{n}\left(\begin{array}{c}
M \\
n
\end{array}\right) \frac{(M+n) !}{(M+n+j) !}\left(\Theta+\frac{1}{2}\right)^{M+n+j}, \\
T_{j}=T_{j}^{(M)} \equiv \sum_{n=0}^{j-1} \frac{H_{j-n}^{(M)}\left(\frac{1}{2}\right)}{n !}\left(\Theta-\frac{1}{2}\right)^{n},
\end{array}\right.
$$

where $\Theta_{a}=-\frac{1}{2}$ and $\Theta_{b}=\frac{1}{2}$

Acknowledgments. The author is indebted to E. Pelinovsky and I. Didenkulova for drawing his attention to the topic at hand. Colleagues K. Mardal, U. Fjordholm and C. Pedersen are thanked for their contributions. Finally, the author is grateful for the valuable suggestions from the anonymous referees of the SIAP.

\section{REFERENCES}

[1] K. Attenborough, K. M. Li, And K. Horoshenkov, Predicting Outdoor Sound, CRC Press, 2006.

[2] V. M. Babic And V. S. Buldyrev, Short-Wavelength Diffraction Theory, Springer Series on Wave Phenomena, Springer-Verlag Berlin Heidelberg, 1972.

[3] S. BASKAR AND P. PRASAD, Propagation of curved shock fronts using shock ray theory and comparison with other theories, Journal of Fluid Mechanics, 523 (2005), p. 171198, https://doi.org/10.1017/ S0022112004001910.

[4] L. M. Brekhovskikh, Waves in layered media, vol. 328 of Applied mathematics and mechanics, Academic Press, second ed., 1980.

[5] B. H. Choi, E. Pelinovsky, D. C. Kim, I. Didenkulova, and S.-B. Woo, Two- and three-dimensional computation of solitary wave runup on non-plane beach, Nonlin. Processes Geophys., 15 (2008), pp. 489-502.

[6] I. Didenkulova And E. Pelinovsky, Non-dispersive traveling waves in inclined shallow water channels, Physics Letters A, 373 (2009), p. 38833887.

[7] I. Didenkulova And E. Pelinovsky, Nonlinear wave evolution and runup in an inclined channel of a parabolic cross-section, Physics of Fluids, 23 (2011), p. 086602. 
[8] I. Didenkulova And E. Pelinovsky, Runup of tsunami waves in u-shaped bays, Pure Appl. Geophys., 168 (2011), p. 12391249.

[9] I. Didenkulova And E. Pelinovsky, On shallow water rogue wave formation in strongly inhomogeneous channels, Journal of Physics A: Mathematical and Theoretical, 49 (2016), p. 11pp, https://doi.org/ 10.1088/1751-8113/49/19/194001.

[10] I. Didenkulova, E. Pelinovsky, And T. Soomere, Long surface wave dynamics along a convex bottom, J. Geophys. Res., 114 (2009), pp. 2156-2202.

[11] I. I. Didenkulova And E. N. Pelinovsky, Reflection of a long wave from an underwater slope, Oceanology, 51 (2011), pp. 568-573, https://doi.org/10.1134/S0001437011040060.

[12] I. I. Didenkulova, N. Zahibo, and E. N. Pelinovsky, Reflection of long waves from a nonreflecting bottom profile, Fluid Dyn., 43 (2008), pp. 590-595.

[13] M. W. Dingemans, Water wave propagation over uneven bottoms. Part 1 - linear wave propagation., vol. 13 of Advanced Series on Ocean Engineering., World Scientific Publishing, Singapore, 1997.

[14] R. GRImshaw, The solitary wave in water of variable depth. part 2, Journal of Fluid Mechanics, 46 (1971), pp. 611-622.

[15] R. Grimshaw, D. Pelinovsky, and E. Pelinovsky, Homogenization of the variable-speed wave equation, Wave Motion, 47 (2010), pp. 496-507.

[16] K. Ko And H. H. Kuehl, Korteweg-de Vries Soliton in a Slowly Varying Medium, Phys. Rev. Let., 40 (1978), pp. 233-236.

[17] K. Ko And H. H. Kuehl, Cylindrical and spherical Korteweg-deVries solitary waves, Phys. Rev. Let., 22 (1979), pp. 1343-1348.

[18] H. Lamb, Hydrodynamics, Cambridge University Press, 6th ed., 1994.

[19] C. C. MEI, The Applied Dynamics of Ocean Surface Waves, World Scientific, 1989.

[20] J. W. Miles, Diffraction of solitary waves, Z. angew. Math. Phys., 28 (1977), pp. 889-902.

[21] J. W. Miles, Solitary waves, Ann. Rev. Fluid Mech., 12 (1980), pp. 11-43.

[22] G. Pedersen, Nonlinear modulations of solitary waves, J. Fluid Mech., 267 (1994).

[23] G. K. Pedersen, Fully nonlinear boussinesq equations for long wave propagation and run-up in sloping channels with parabolic cross sections, Natural Hazards, 84 (2016), pp. 599-619. DOI:10.1007/s11069016-2448-0.

[24] E. Pelinovsky, I. Didenkulova, E. Shurgalina, and N. Aseeva, Nonlinear wave dynamics in selfconsistent water channels, J. Phys. A: Math. Theor., 50 (2017), pp. 285-311.

[25] D. H. Peregrine, Solitary waves in trapezoidal channels, J. Fluid Mech., 35 (1969), pp. 1-6.

[26] A. S. Peters, Rotational and irrotational solitary waves in a channel with arbitrary cross, Communications on Pure and Applied Mathematics, XIX (1966), pp. 445-471.

[27] E. M. Salomons, Computational Atmospheric Acoustics, Kluwer Academic Publishers, 2001.

[28] M. C. Shen, Asymptotic theory of unsteady three-dimensional waves in a channel of arbitrary cross section, SIAM Journal on Applied Mathematics, 17 (1969), pp. 260-271.

[29] T. Shimozono, Long wave propagation and run-up in converging bays, Journal of Fluid Mechanics, 798 (2016), p. 457484, https://doi.org/10.1017/jfm.2016.327.

[30] C. E. Synolakis, The run-up of solitary waves, J. Fluid Mech., 185 (1987), pp. 523-545.

[31] S. Tadepalli And C. Synolakis, The run-up of N-waves on sloping beaches, Proc. R. Soc. Lond. A, 445 (1994), pp. 99-112.

[32] W. C. Thacker, Some exact solutions to the nonlinear shallow-water wave equations, J. Fluid Mech., 107 (1981), pp. 499-508.

[33] S. Tinti, E. Bortolucci, And C. Chiavettieri, Tsunami Excitation by Submarine Slides in Shallowwater Approximation, Pure and Applied Geophysics, 158 (2001), pp. 759-797.

[34] G. B. Whitham, A new approach to problems of shock dynamics part i two-dimensional problems, Journal of Fluid Mechanics, 2 (1957), p. 145171, https://doi.org/10.1017/S002211205700004X.

[35] G. B. Whitham, Linear and nonlinear waves, Pure \& Applied Mathematics, John Wiley \& sons, New York, 1974. 